\title{
Comportamiento epidemiológico de la morbilidad materna extrema. Colombia, 2016
}

\section{Erika T Sánchez-Barrera', Sandra P Mendieta-Hernández², Eyleen M Pineda-Martínez, Deysi L Cárdenas-Pinzón ${ }^{4}$}

\section{RESUMEN}

Introducción. La Morbilidad Materna Extrema (MME) es un problema de salud pública porque compromete la vida de la gestante. Esta complicación obstétrica también pone en riesgo la salud y el bienestar del recién nacido y de los hijos ya existentes.

Objetivo. Determinar el comportamiento epidemiológico de la MME en Colombia durante el año 2016.

Materiales y métodos. Estudio descriptivo tipo ecológico en mujeres entre 10 a 59 años, residentes en Colombia y con historial médico de MME durante el embarazo, el parto o el puerperio. Los datos se obtuvieron del Sistema Nacional de Vigilancia en Salud Pública (SIVIGILA) y el Sistema Integral de Información de la Protección Social (SISPRO). Se calculó el indicador de carga de enfermedad Años de Vida Perdidos por Discapacidad (AVPD), mediante herramientas empleadas por la Organización Mundial de la Salud.

Resultados. El mayor número de AVPD por MME se presentó en el grupo de 15 a 29 años con un total de 5,86 AVPD. Se evidencia que Bogotá suma 1,93 AVPD a la carga de MME en el país.

Conclusión. La MME tiene mayor prevalencia en la capital del país, aunque el 67,2\% de los casos notificados se encuentran en los departamentos con mayor nivel de complejidad de atención en salud y capacidad de respuesta de calidad.

Palabras clave: epidemiología, morbilidad materna, discapacidad.

\footnotetext{
${ }^{1}$ Comparta EPS, Tunja, Colombia.

${ }^{2}$ Secretaría de Salud de Boyacá, Tunja, Colombia.

${ }^{3}$ Hospital Santa Rosa de Viterbo, Santa Rosa de Viterbo, Colombia.

${ }^{4}$ Universidad de Boyacá, Tunja, Colombia
}

Correspondencia: Deysi L Cárdenas-Pinzón Dirección Carrera 2a este № 64-169. Tunja (Boyacá), Colombia. Celular: 3214711189

Correo electrónico: deycardenas@uniboyaca.edu.co

Citar este artículo así:

Sánchez Barrera ET, Mendieta Hernández SP, Pineda Martínez EM, Cárdenas Pinzón DL. Comportamiento epidemiológico de la morbilidad materna extrema. Colombia, 2016. Revista Investig Salud Univ Boyacá. 2019;6(2): 99-117. doi: https://doi.org/10.24267/23897325.414 


\title{
Epidemiological behavior of extreme maternal morbidity. Colombia, 2016
}

\begin{abstract}
Introduction. Extreme Maternal Morbidity (MME) is a public health problem that compromises the life of the pregnant woman. This obstetric complication also puts the health and well-being of the newborn and the children already at risk.
\end{abstract}

Objective. To determine the epidemiological behavior of the MME in Colombia during 2016.

Materials and methods. Ecological descriptive study in women aged 10 to 59 years, residents in Colombia and medical history of MME during pregnancy, delivery or puerperium. The data were obtained from the National System of Surveillance in Public Health (SIVIGILA) and the Integral Information System of Social Protection (SISPRO). The indicator of the burden of the disease Years of life Lost due to Disability (AVPD) was calculated using tools used by the World Health Organization.

Results: The highest number of AVPD per MME was presented in the group of 15 to 29 years with a total of 5.86 AVPD. It is evident that Bogota adds 1.93 AVPD to the Load of MME in the country.

Conclusion. MME has a higher prevalence in the country's capital, although $67.2 \%$ of cases are found in the departments with the highest level of health care and quality response capacity.

Keywords: epidemiology, maternal morbidity, near miss, disability. 


\title{
Comportamento epidemiológico da morbidade materna extremamente grave Colômbia, 2016
}

\begin{abstract}
RESUMO
Introdução. A morbidade Materna Extremamente Grave (MMEG) é um problema de saúde pública porque compromete a vida da mulher grávida. Esta complicação obstétrica também coloca em risco a saúde e o bem estar do recém-nascido e dos filhos existentes.
\end{abstract}

Objetivo. Determinar o comportamento epidemiológico da MMEG na Colômbia em 2016.

Materiais e métodos. Estudo descritivo do tipo ecológico em mulheres entre 10 e 59 anos, residentes na Colômbia e com histórico médico de MMEG durante a gravidez, parto ou puerpério. Os dados foram obtidos no Sistema Nacional de Vigilância em Saúde Pública (SIVIGILA) e no Sistema Integral de Informação da Proteção Social (SISPRO). O indicador carga da doença Anos de Vida Perdidos por Incapacidade (YLD) foi calculado, através de ferramentas utilizadas pela Organização Mundial da Saúde.

Resultados. O número elevado de YLD por MMEG aparece no grupo entre 15 e 29 anos com um total de 5,86 YLD. É evidente que Bogotá acrescenta 1,93 YLD à carga de MMEG no país.

Conclusão. A MMEG tem uma prevalência mais alta na capital do país, embora o $67,2 \%$ dos casos relatados estão nos departamentos com um nível mais alto de complexidade de atenção e capacidade de resposta de qualidade.

Palavras-chave: epidemiologia, morbidade materna, Incapacidade. 


\section{INTRODUCCIÓN}

La Morbilidad Materna Extrema (MME) es definida por la Organización Mundial de la Salud (OMS) como un episodio que potencialmente amenaza la vida de una mujer debido a una complicación grave durante el lapso de la gestación, el parto o los 42 días después del parto (1). La vigilancia y seguimiento de este evento es de suma importancia dado que informa y da idea del grado de desarrollo económico de un país, del alcance de su infraestructura y la calidad de sus servicios de salud.

En los últimos años, los casos relacionados con MME han tenido un creciente interés a nivel mundial, pues se hace necesario encontrar estrategias preventivas de la mortalidad materna (MM) y perinatal (MP). En este sentido, lo que se busca en los diferentes países es la atención oportuna y adecuada a una mujer con complicación obstétrica extrema con el fin de prevenir una discapacidad permanente o un desenlace fatal.

En 1990 se reportó a nivel global una tasa de mortalidad materna de 385 por cada 100,000 nacidos vivos. Más adelante, en el año 2015, se reportó una tasa de mortalidad materna de 216 por cada 100,000 nacidos vivos, lo que evidenció una reducción, aunque no la esperada (2). Se estima que el $90 \%$ de estas muertes son evitables mediante prácticas seguras y preventivas, lo cual es posible de llevar a cabo en todos los países del mundo.
Cada año, aproximadamente 300 millones de mujeres padecen patologías o discapacidades conexas con la gestación, el parto o el posparto, como también se considera que cada año hay alrededor de 50 millones de complicaciones en salud materna $(3,4)$. Por lo anterior, la Organización Panamericana de la Salud (OPS) y la OMS propusieron realizar vigilancia epidemiológica de la MME como estrategia adicional para la prevención, control e identificación oportuna de factores determinantes, que permitieran lograr la reducción de las tasas de MM en tres cuartas partes.

Esta estrategia se realiza como un pilar importante en el desarrollo de la salud pública de todos y cada uno de los países del mundo, fomentando el interés y la responsabilidad por la salud de los colectivos y las poblaciones como bien público.

En el mundo, las complicaciones de salud durante la gestación y el parto son responsables del 13 al $18 \%$ de enfermedades en mujeres entre los 15 y 44 años, lo cual repercute significativamente en la salud y el bienestar del recién nacido y los hijos ya existentes. Cabe resaltar que cuando las mujeres fallecen por complicaciones durante el embarazo, parto o posparto, los recién nacidos tienen 10 veces más riesgo de morir (5). Además, las madres sobrevivientes pueden generar discapacidades y problemas de salud que van a obstaculizar o limitar el cuidado de los hijos; por tanto, la no vigilancia en salud materna y la no prevención oportuna de los factores de riesgo de 
la misma, influye en gran medida en el desarrollo humano $y$, en esa medida, en el curso evolutivo de las naciones, pues se relaciona con un país en capacidad de desarrollo, con la calidad de desarrollo de sus niños y niñas, y con la capacidad de atención a ello desde la gestación, tal como lo sostienen las cifras a continuación: la tasa de MME es mayor en los países de medianos y bajos ingresos; es así que la tasa de MME en América Latina es de $4,9 \%, 5,1 \%$ en Asia y $14.9 \%$ en África; en contraste, los países de altos ingresos demuestran tasas que van del $0,8 \%$ en Europa al $1,4 \%$ en Norteamérica (6).

Una revisión de MME en Colombia durante el periodo 2003-2006, concluyó que las principales causas por las que se presenta morbilidad materna extrema, son: preeclampsia severa (75\%), hemorragia (34.3\%) y síndrome de HELLP (31.5\%) (7); causas claramente prevenibles desde la identificación de factores de riesgo que permitan controlar la aparición de estas patologías.

En el año 2012 empezó la vigilancia de este evento, por esfuerzos conjuntos del Ministerio de Salud y Protección Social (MinSalud), el Fondo de Población de las Naciones Unidas y el Instituto Nacional de Salud (INS); así, se logró la implementación en el Sistema de Vigilancia Epidemiológica (SIVIGILA) a través de la notificación individual de casos. Según cifras gubernamentales presentadas por el INS, la razón de MME para el año 2012 fue de 6,9 por mil NV, en el año 2013 la razón fue de 13,1 por mil NV, en el año 2014 fue de 17,8 casos por cada mil NV, para el año 2015 se calculó en 64 por cada 100,000 NV, durante el año 2016 se notificaron 20.600 casos que representaron un incremento de $33,7 \%$ con respecto al año anterior.

La razón de MME nacional preliminar fue de 31 casos por 1.000 nacidos vivos, sin embargo, 13 entidades territoriales la superan. Las cinco entidades territoriales con mayor razón de MME preliminar son: Cartagena, con 58,2; Vichada, con 55,7; Huila, con 50,4; La Guajira, con 48,6; y Bogotá, con 46,1 casos por 1.000 nacidos vivos. $(8,9)$.

En Bogotá se encuentra la mayor cantidad de investigaciones sobre MME en Colombia, seguida por ciudades como Cali, Meta, Nariño, Medellín y Cartagena, lo cual evidencia la necesidad de estudios que abarquen no solo la población urbana y de las principales cabeceras municipales, sino que también involucre población rural y comunidades de baja densidad poblacional, de tal forma que se pueda identificar el comportamiento de las complicaciones en todas y cada una de las gestantes colombianas $y$, asimismo, se generen estrategias que permitan reducir la aparición del evento.

En los artículos revisados, se observa una mayoría de estudios transversales (descriptivos, ecológicos, observacionales), otros de casos y controles, y uno mixto (cualitativo-cuantitativo). 
La salud materna es un tema prioritario de salud pública para las autoridades gubernamentales del país, por tanto, se han hecho esfuerzos por incluirlo en normas y políticas como el Plan Decenal de Salud Pública PDSP 2012-2021, que propone lograr la equidad en salud y el desarrollo humano de todos los colombianos mediante ocho dimensiones prioritarias y dos transversales (10) en busca del mejoramiento a largo y mediano plazo de las cifras de morbilidad y mortalidad materna. Aunque en Colombia se han realizado avances significativos, todavía quedan aristas de la problemática que merecen atención. En este orden de ideas, el problema de la morbilidad materna extrema se debe abordar y estudiar para crear conocimiento a su alrededor, además de nuevos datos en términos de años perdidos por discapacidad que aporten a la salud pública de Colombia, teniendo en cuenta que las mujeres que sobreviven a este evento merecen no solo servicios de salud con calidad, eficiencia y calidez, sino talento humano preparado y empoderado en conocimiento e identificación oportuna de factores de riesgo y posibles complicaciones obstétricas, teniendo como premisa que este tipo de eventos influyen en la pérdida de calidad de vida de las mujeres y sus familias. También, se deben tener en cuenta que las condiciones económicas y sociales de una mujer sobreviviente con discapacidad deben ser favorables para el desarrollo de su vida en todos los aspectos, pues no solo afecta su rol materno, sino también su vida en general y su progreso social efectivo.
Finalmente, los estudios encontrados sirven para comprender de una manera más amplia y clara los factores de riesgo y las causas predisponentes de la MME, además, ofrecen herramientas a los profesionales de la salud para identificarlos a tiempo y brindar una atención de calidad y oportuna a las gestantes $y$, de esta manera, asegurarle una mejor calidad de vida al producto de su gestación, para garantizar el desarrollo de un nuevo integrante de la sociedad, productivo para la misma y con capacidad de aportar, en gran medida, a su evolución.

También se puede evidenciar por medio de la revisión literaria, que la MME se presenta de forma alarmante, razón por la cual son necesarias diversas investigaciones que permitan unificar conceptos, abordar de manera integral a la población, mejorar las redes de apoyo, identificar poblaciones vulnerables o en riesgo, identificar factores predisponentes que se puedan minimizar $y$, en general, ir un paso adelante en la aparición de patologías en la gestación, para brindar una atención de calidad a todas y cada una de las mujeres en etapa de gestación, parto y posparto, de tal forma que se logren disminuir las tasas de MME.

\section{MATERIALES Y MÉTODOS}

A través de un estudio de tipo ecológico exploratorio se describió el comportamiento epidemiológico de la Morbilidad Materna Extrema (MME) 
en mujeres residentes en Colombia, entre los 10 y 59 años, que presentaron algún episodio que potencialmente amenazó sus vidas, es decir, sobrevivieron a una complicación ocurrida durante el embarazo, el parto o los 42 días de puerperio durante el año 2016 (1). En primer lugar, se estimó la población obteniendo los datos del cubo SIVIGILA del Sistema Integral de Información de la Protección Social (SISPRO).

La población está constituida por la totalidad de los casos presentados durante el 2016 y reportados por las Unidades Primarias Generadoras de Datos (UPGD) al Instituto Nacional de Salud (INS). Las mujeres incluidas en el estudio fueron sobrevivientes de una complicación obstétrica. Se excluyeron mujeres por fuera del rango de edad definido o que fallecieron y fueron notificadas como Mortalidad Materna, con patologías relacionadas con otros eventos como violencia, enfermedades propias de base, entre otras.

En la construcción de indicadores, objetivo de esta investigación, se usaron los datos del Departamento Administrativo Nacional de Estadísticas (DANE) para identificar la población por rangos de edad. Además, el proyecto buscó estimar los Años de Vida Perdidos por Discapacidad (AVPD), un indicador de carga de enfermedad contemplado en la herramienta "The Global Burden of Disease study" (11-13) de la Organización Mundial de la Salud (OMS).
Para realizar la medición se generaron 10 grupos de edad. El indicador de carga de la enfermedad evaluado fue Años de Vida Perdidos por Discapacidad (AVPD), que es el resultado de multiplicar el número de casos por el peso de discapacidad para el evento; de esta manera, se representa la pérdida de un año completamente saludable. Para el cálculo de ese indicador se usaron: la incidencia de la MME, duración de la enfermedad, promedio de edad de inicio de la enfermedad para cada grupo de edad escogido y, finalmente, se tuvo en cuenta el peso de la discapacidad. Bedregal (14) sostiene que "el peso de la discapacidad corresponde a la asignación de un valor único, que expresa el juicio social cuantificado, que se otorga al estado de salud actual de un sujeto, sobre el curso de la enfermedad" (p. 28).

De acuerdo con lo anterior, se utilizó un enfoque basado en la medición de discapacidad, a través de la clasificación internacional de impedimentos y discapacidad de la OMS para las causas relacionadas con MME, teniendo en cuenta los criterios utilizados para establecer los pesos correspondientes a MME $(13,15)$. Este estudio fue avalado por el Comité de Bioética de la Universidad de Boyacá, según memorando CB 438 del 26 de julio de 2018. 
Tabla 1. Distribución de la MME por departamento y rango de edad

\begin{tabular}{|c|c|c|c|c|c|c|c|c|c|c|}
\hline $\begin{array}{c}\text { Departamento / Rango } \\
\text { de edad }\end{array}$ & $10-14$ & $15-19$ & $20-24$ & $25-29$ & $30-34$ & $35-39$ & $40-44$ & $45-49$ & $50-54$ & $55-59$ \\
\hline Antioquia & 17 & 291 & 403 & 320 & 245 & 179 & 54 & 8 & & \\
\hline Atlántico & 15 & 279 & 313 & 236 & 167 & 114 & 33 & 1 & 1 & \\
\hline Bogotá, D.C. & 22 & 569 & 1.094 & 1.120 & 1.028 & 717 & 220 & 21 & 1 & \\
\hline Bolívar & 33 & 421 & 507 & 376 & 296 & 167 & 43 & 6 & & 1 \\
\hline Boyacá & 1 & 66 & 81 & 80 & 104 & 49 & 22 & 2 & & \\
\hline Caldas & 2 & 27 & 51 & 38 & 22 & 20 & 5 & & & \\
\hline Caquetá & 4 & 45 & 35 & 22 & 26 & 16 & 9 & & & \\
\hline Cauca & 13 & 141 & 150 & 107 & 93 & 66 & 33 & 2 & & \\
\hline Cesar & 6 & 111 & 155 & 135 & 88 & 58 & 22 & 3 & & \\
\hline Córdoba & 16 & 176 & 248 & 167 & 159 & 104 & 32 & 4 & & \\
\hline Cundinamarca & 10 & 241 & 310 & 279 & 241 & 156 & 52 & 2 & 1 & \\
\hline Chocó & 3 & 13 & 28 & 20 & 12 & 7 & 5 & & & \\
\hline Huila & 6 & 264 & 235 & 199 & 153 & 111 & 42 & 6 & & 1 \\
\hline La Guajira & 21 & 150 & 158 & 137 & 106 & 61 & 32 & 4 & & \\
\hline Magdalena & 14 & 240 & 226 & 190 & 147 & 82 & 20 & 4 & & \\
\hline Meta & 6 & 75 & 89 & 67 & 49 & 31 & 7 & 1 & & \\
\hline Nariño & 5 & 102 & 114 & 116 & 91 & 67 & 27 & 1 & & \\
\hline Norte de Santander & 4 & 67 & 63 & 65 & 48 & 45 & 17 & 2 & & \\
\hline Quindio & 1 & 16 & 23 & 20 & 15 & 16 & 2 & 2 & & \\
\hline Risaralda & 1 & 26 & 44 & 45 & 26 & 23 & 7 & & & \\
\hline Santander & 5 & 95 & 128 & 106 & 79 & 56 & 27 & 4 & & \\
\hline Sucre & 3 & 62 & 63 & 50 & 35 & 31 & 11 & & & 1 \\
\hline Tolima & 1 & 97 & 106 & 77 & 67 & 39 & 16 & 1 & & \\
\hline Valle del Cauca & 8 & 179 & 313 & 308 & 231 & 165 & 45 & 7 & & 1 \\
\hline Arauca & 5 & 53 & 35 & 17 & 22 & 11 & 2 & . & & \\
\hline Casanare & 4 & 51 & 52 & 35 & 30 & 31 & 8 & 2 & & \\
\hline Putumayo & 1 & 29 & 31 & 23 & 19 & 14 & 6 & 1 & & \\
\hline Archipiélago de San An & & 3 & 4 & 8 & 2 & & 1 & & & \\
\hline Amazonas & & 9 & 7 & 6 & 5 & 7 & 1 & & & \\
\hline Guainía & & 4 & 7 & 2 & 1 & 1 & & & & \\
\hline Guaviare & & 5 & 6 & 1 & 2 & 4 & & & & \\
\hline Vaupés & & & 1 & 5 & 1 & 1 & & & & \\
\hline Vichada & 1 & 13 & 17 & 10 & 9 & 7 & 2 & & & \\
\hline
\end{tabular}

Fuente: SIVIGILA, SISPRO 2016

\section{RESULTADOS}

El presente estudio tomó como población objeto mujeres en estado de gestación que presentaron complicaciones durante su proceso de parto o puerperio. Dicha información se extrajo de las bases de datos del Instituto Nacional de Salud (SIVIGILA-SISPRO), entes territoriales para la vigilancia epidemiológica de eventos de interés en salud pública. Estos datos corresponden a la 
población colombiana específicamente discriminada por departamentos y rangos de edad de las gestantes.

La tabla 1 muestra los casos de Morbilidad Materna Extrema presentados en los diferentes departamentos de Colombia, divididos por rangos de edad; como se puede observar, los departamentos de Antioquia, Atlántico, Bogotá, Bolívar, Córdoba, Cundinamarca, Huila, Magdalena y Valle del Cauca son los que mayor cantidad de casos de MME presentan. De igual forma, se evidencia que los rangos de edad en los que se presentan estos casos son de 15-29 años y 30-44 años.

En la figura 1 se observa que Bogotá, al ser la capital de Colombia, reporta 1,93 AVPD, seguida por Bolívar, Antioquia, Cundinamarca y Valle del Cauca, cuyo reporte es de 0,73, 0,61, 0,52, 0,50, respectivamente.

Figura 1. Años de Vida perdidos por Discapacidad por departamento

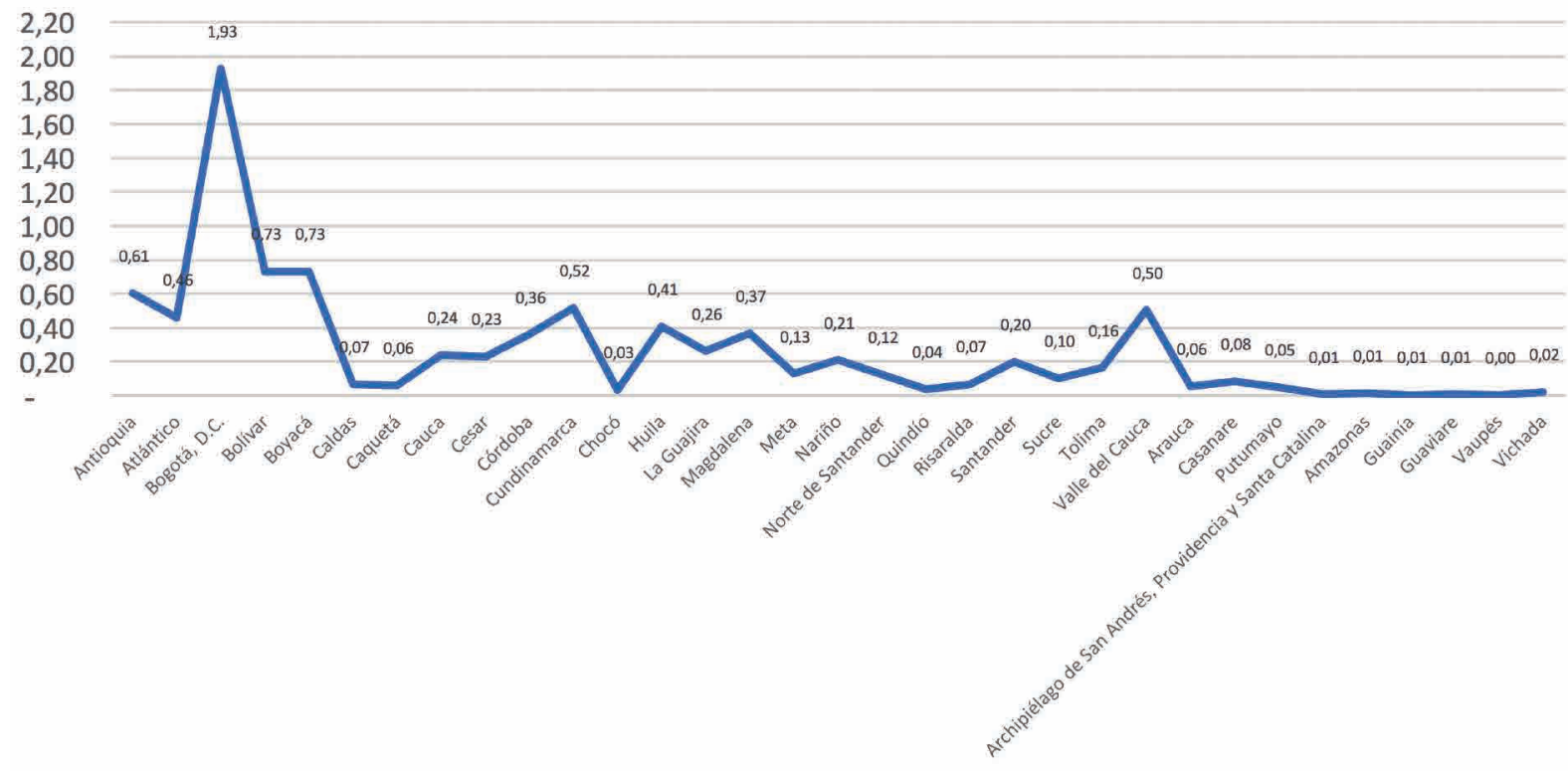

Fuente: SIVIGILA, SISPRO 2016 
En la tabla 2 se puede observar que el rango de edad en el cual se presentó la mayor cantidad de casos de MME fue de 15 a 29 años con un total de 5,86 AVPD; seguido por el rango de 30 a 44 años con 2,92 AVPD; en tercer lugar, encontramos el rango de edad de 45 a 59 años con 0,02 AVPD.

Tabla 2. Años de Vida perdidos por Discapacidad por Rangos de Edad

\begin{tabular}{cc}
\hline Rango de edad & AVPP \\
\hline $5-14$ & 0 \\
\hline $15-29$ & 5,86 \\
\hline $30-44$ & 2,92 \\
\hline $45-59$ & 0,02 \\
\hline
\end{tabular}

Fuente SIVIGILA, SISPRO2016

En la figura 2 se evidencia que el mayor número de casos fue notificado en Bogotá D.C., cifra esperada por tratarse de una ciudad donde se concentra la mayoría de Instituciones Prestadoras de Salud (IPS) con mayor nivel de complejidad de atención y que, además, son referencia de capacidad técnico científica para brindar los servicios que ameritan las mujeres que presentan MME. Sin embargo, la gráfica 3 solamente da cuenta del departamento de residencia real de la mujer, mas no del departamento donde fue atendida. Teniendo en cuenta lo anterior, las entidades territoriales que aportaron mayor número de casos a las cifras nacionales fueron: Bogotá (4792), Bolívar (1850), Antioquia (1.517), Cundinamarca (1292), Valle del Cauca (1257), Atlántico (1159), Huila (1017), Córdoba (906) y la Guajira (669): estas cifras representan el $67,2 \%$ de los casos notificados en el país durante el año 2016. Cabe resaltar que estos departamentos tienen IPS con mayor nivel de complejidad de servicios de salud, menos dificultades para el acceso a estos servicios, en contraposición a los departamentos que las autoras esperaban que tuvieran mayoría de casos por sus dificultades demográficas, sociales y económicas, como el Chocó y el Amazonas, los cuales no encabezan la lista y, de hecho, presentan pocos casos, 88 y 35, respectivamente, durante el año 2016. Sin embargo, es necesario ahondar en el tema con razón de MME.

\section{DISCUSIÓN}

En Colombia, durante el año 2016, la Morbilidad Materna Extrema mostró un incremento significativo del $33,7 \%$ en la tasa de morbilidad con respecto al año 2015, en que se calculó 64 por cada 100.000 nacidos vivos (NV) $(8,9)$. Dicho comportamiento también se refleja a nivel internacional, según una investigación ejecutada por Cuesta, en México, durante el año 2017 (7). Allí, señala una gran diferencia de las frecuencias de MME: 165 casos por año en 2017 y 20.600 casos por año en 2016. 
Figura 2. Desigualdades por departamento - Notificación por entidad territorial de residencia.

$\square$ MME $\square$ MEDIANA $\%$

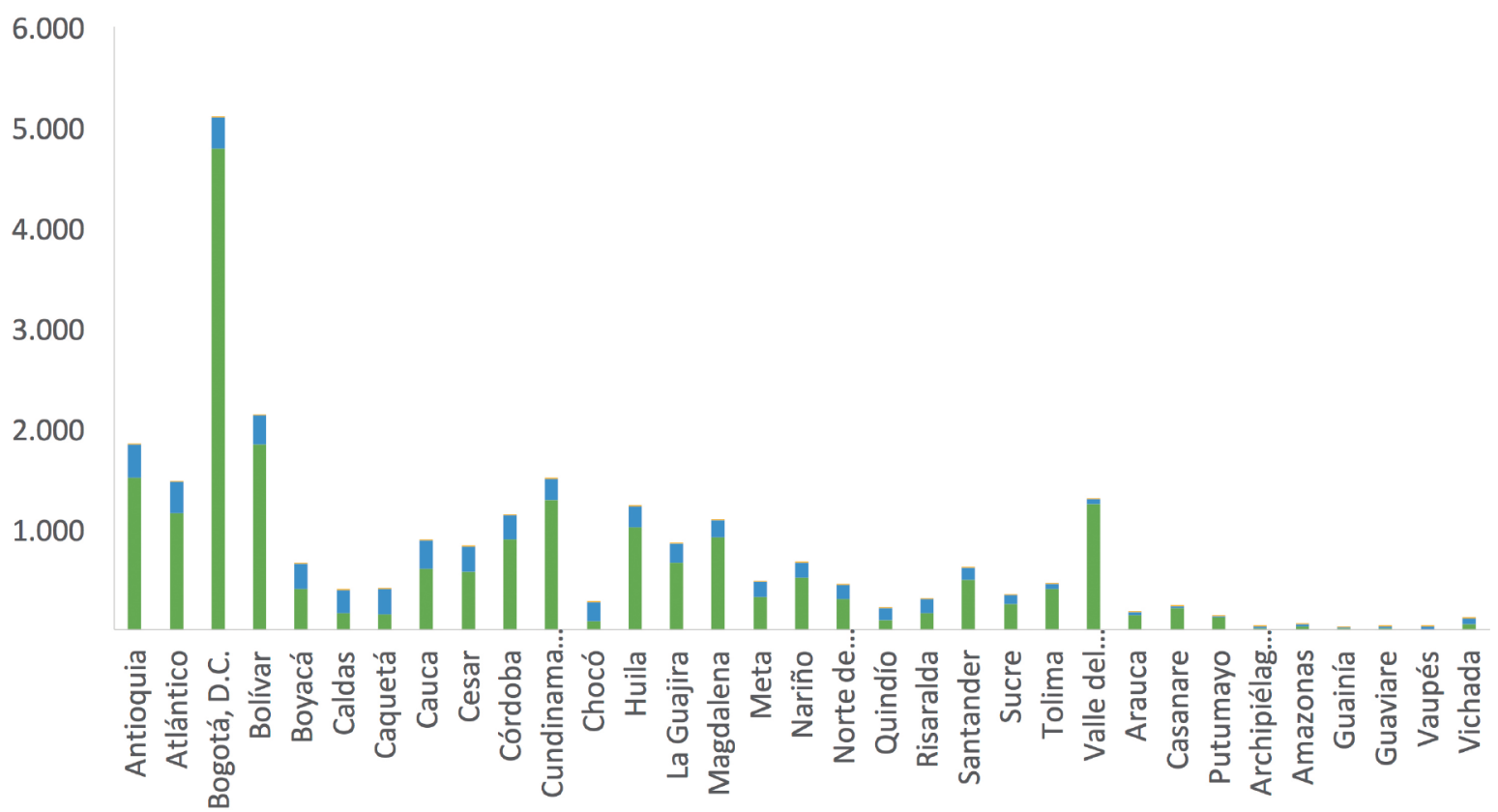

Fuente: SIVIGILA, SISPRO 2016

Cabe resaltar que el actual estudio muestra que la mayor cantidad de casos de MME se presenta en los departamentos de Antioquia, Atlántico, Bogotá, Bolívar, Córdoba, Cundinamarca, Huila, Magdalena y Valle del Cauca, donde figura Bogotá como la ciudad con mayor índice de AVPP $(1,93)$ debido a que, posiblemente, allí se concentran la mayoría de Instituciones Prestadoras de Salud (IPS) con mayor nivel de complejidad de atención, que son referencia de capacidad técnico científica para brindar los servicios que ameritan las mujeres con MME.

En cuanto a los demás departamentos, se presentaron los siguientes reportes de AVPP: Bolívar 
$(0,73)$, Antioquia $(0,61)$, Cundinamarca $(0,52)$, Valle del Cauca $(0,50)$. Así mismo, los rangos de edad de estos casos figuran entre los 15-29 años y los 30-44 años. En Colombia, para el año 2016, hubo 5,86 AVPD en el rango de 15 a 29 años, seguido por 2,92 AVPD en el rango de 30 a 44 . Al cotejar estos resultados con los encontrados en los estudios realizados a nivel individual por cada departamento, se constata que la MME en el transcurso del tiempo ha tenido un incremento notable, lo cual revela que se deben fortalecer estos programas a fin de mejorar la atención en salud para las mujeres en estado de gestación, parto y puerperio.

De la misma manera, se puede evidenciar que en la mayoría de las investigaciones revisadas hay una alta incidencia en las principales causas y complicaciones de la MME, lo que manifiesta un aumento significativo en la frecuencia de la MME. Un ejemplo de esto es el estudio de estimación de carga de enfermedad del año 2010, elaborado por Peñalosa (16), en el cual se menciona la prevalencia/incidencia de las diferentes complicaciones durante la gestación, parto y puerperio; además, agrega que a nivel mundial, en el periodo 2008-2010, se presentó una tasa de incidencia de trastorno hipertensivo del $11 \%$ y para la hemorragia materna y obstétrica del $11.2 \%$.

Por otro lado, la investigación de Castañeda et al (17) sostiene que la complicación más relevante es la relacionada con los Trastornos Hipertensivos Asociados en el Embarazo (THAE) como la tensión arterial con $31,4 \%$, seguida por la preeclamsia con el 16,6\%; esto demuestra cuáles son los padecimientos que más generan discapacidades y fallecimientos en las gestantes. El artículo elaborado por Gonzáles et al en el año 2015 (18) señala que la tasa de mortalidad y morbilidad materna fue de 11,1 por cada $100 \mathrm{NV}$ y 5,6 por cada 1000 NV. También, Bendezú (19) afirma que la razón de morbilidad es de 3,57 por 1000 NV, con un índice de mortalidad de 6,1.

En Colombia, el estudio realizado por Barbosa et al $(20,21)$ en Cali, para el año 2016, refleja un incremento significativo de casos de MME: 219 durante los años 2013 y 2014. En cambio, los resultados obtenidos en esta investigación revelan que la frecuencia de la MME estuvo representada en 1.257 casos aproximadamente. Es relevante destacar las variables que se tuvieron en cuenta, como la edad, cuyo promedio fue de 26 años; así mismo, la complicación más frecuente fue la de los THAE, con un $74,4 \%$. Villalba et al (22) afirma que en Bogotá la prevalencia de MME es de 11 por $1000 \mathrm{NV}$, lo cual prueba que para el año 2013 las cifras eran bajas con respecto al año estudiado, 2016. En los casos reportados, el 55\% correspondió a complicaciones relacionadas con hemorragias del embarazo y los THAE. 
En el contexto del departamento del Meta, CarriIlo (23) señala que el número de casos en el año 2014 fue de 353 con respecto al año anterior, con 194 casos. Aquí se consideraron el promedio de edad de 25,2 años y el primer factor de riesgo como la eclampsia con el $22,38 \%$. Por otro lado, Salazar (3) da a conocer 1384 casos en total en Medellín, presentados en edades comprendidas entre los 20 y 29 años. Con esto se expone que la razón de MME para los años 2013- 2015 fue de 17,7-13,0-18,0 por $1000 \mathrm{NV}$, resultado que coincide con el obtenido en esta investigación, sobre el grupo de edad más afectado por MME.

Donoso et al (24) buscó determinar el quinquenio de edad materna con menor riesgo de complicaciones en el embarazo y mortalidad; en este sentido, concluyó que el grupo de mujeres mayores de 35 años es el más afectado por las complicaciones obstétricas $\mathrm{y}$, por tanto, tiene mayor riesgo de presentar morbilidad materna extrema. Esta misma conclusión coincide con la de Huang (25), quien por medio de una revisión sistemática evidenció mayor riesgo en este grupo etario. Por su parte, Kenny (26) demostró en un estudio de cohorte retrospectivo, con datos del año 2004 al 2008 en Singleton (Reino Unido), que el riesgo de una amplia gama de desenlaces perinatales y maternos adversos incrementan con el aumento de la edad materna. Estos resultados, sin embargo, no coinciden exactamente con la información obtenida en el presente estudio, debido a que, como se mencionó, el grupo de edad con mayor número de casos fue de 15-29 años, seguido del grupo de gestantes de 30-44 años. Estas diferencias responden también a la forma en que cada estudio construye los grupos de edad. Para la presente investigación se tuvo en cuenta lo establecido para medir pesos de discapacidad por la OMS.

Es preciso enfatizar que en este estudio el $67,2 \%$ de los casos de MME notificados en el país, durante el año 2016, se concentra en 9 departamentos con las ciudades más desarrolladas en Colombia, es decir, con políticas para reducir comorbilidades previas y durante la gestación, con mayor acceso a atención médica calificada, con aumento de programas educativos alrededor de la gestación, donde se fomenta la atención del parto en instituciones de salud idóneas, en suma, ciudades que cuentan con reducción en la tasa de fecundidad total (7). Así mismo, se resalta un panorama del evento en el país, donde se identifican desigualdades en los territorios; sin embargo, aquellas entidades territoriales donde no se presentan casos o están por debajo de la media, no necesariamente están exentas de casos de MME porque es un evento que se encuentra aún en proceso de investigación por parte del Instituto Nacional de Salud (INS) y es probable que algunos casos no se hayan identificado ni se hayan notificado al Sistema de Vigilancia Epidemiológica, en especial, aquellos ocurridos en comunidades aisladas y comunidades indígenas. 
Según Murray (27), en la actualidad los estudios de carga de enfermedad son herramientas de gran valor en la epidemiología porque los AVPD son indicadores de carga de enfermedad que valoran el impacto y las secuelas de diversos estados de salud; elementos que no se tienen en cuenta en el cálculo de medidas convencionales de morbilidad y mortalidad. Al considerar este marco se logró medir el índice de los AVPD de la MME, y se evidenciaron los siguientes por departamento: Bogotá (1,93 AVPD), Bolívar (0,73 AVPD), Antioquia (0,61 AVPD), Cundinamarca (0,52 AVPD), Valle del Cauca (0,50 AVPD) y, en menor proporción, los departamentos de San Andrés, Providencia y Santa Catalina, Amazonas, Guainía, Guaviare y Vaupés (0,01 y 0,00 AVPD). Además, se puede señalar que el rango de edad que contribuyó para los Años de Vida Perdidos por Discapacidad fue de 15 a 29 años, seguido de 30 a 44 años.

Cabe subrayar la poca información sobre carga de enfermedad por MME en los países de Latinoamérica, lo cual dificulta la comparación de los resultados de este estudio con la situación de naciones con contextos similares al de Colombia. Por otro lado, las publicaciones citadas que corresponden a otros países se enfocan en la descripción de las causas y factores de riesgo para la Morbilidad Materna Extrema, pero no sobre carga de dicho evento en la salud de la población.
En cuanto a las desigualdades en los AVPD en la carga del evento en Colombia, no existen estudios con los que se puedan confrontar y discutir las cifras AVPD de esta investigación; sin embargo, es importante recalcar que Bogotá tiene las cifras más elevadas de MME, tanto por residencia como por lugar de atención, además de tener la mayor proporción de AVPD $(1,93)$. Esto resulta interesante ya que en otros estudios de carga de enfermedad realizados en Colombia sobre otros eventos, como el de Castañeda C (28) sobre la leucemia, no ocurre lo mismo, sino que los AVPD son menores en ciudades con alta capacidad humana y tecnológica, lo que favorece menor discapacidad y mayor posibilidad de recuperación y calidad de vida.

Así mismo, es preciso señalar que, como ya se informó, los menores índices de AVPD se presentaron en departamentos como Chocó, Amazonas, Guainía, Guaviare y Vaupés. Sin embargo, podría haber un sesgo en las desigualdades expuestas en este estudio por departamento de procedencia, teniendo en cuenta que un recién nacido y la madre que ha pasado por Morbilidad Materna Extrema y sus secuelas, pueden requerir varios meses de atención médica. Con respecto a lo anterior, una larga estadía en un lugar se puede entender también como un cambio de residencia. Además, en estos departamentos es mayor el sub-registro en la notificación, las barreras geográficas y de desplazamiento para adquirir servicios 
adecuados y oportunos de salud materna y adherencia a tratamientos durante la gestación. Esto hace pensar que la cifra en estos departamentos podría ser mayor.

Respecto a las limitaciones de este estudio, es preciso mencionar el difícil acceso a las bases de datos de Morbilidad Materna Externa en Colombia, así como la insuficiencia de recursos para desarrollar publicaciones sobre el comportamiento epidemiológico específico de MME. Como fortaleza, este estudio identifica claramente los grupos de edad donde más se presentan los casos, así como los AVPD, generados por las complicaciones de este evento, lo cual es fundamental para generar conocimiento local, efectuar y robustecer estrategias que contribuyan a reducir la morbilidad materna extrema.

Por último, se hace un llamado sobre la importancia de continuar con estudios profundos sobre el tema en comunidades indígenas y/o vulnerables y aisladas en el país. Otros enfoques propuestos son aquellos que abarquen las causas más relevantes de este evento y/o el estado de los hijos recién nacidos de las mujeres que pasan por una complicación obstétrica, así como la carga de la madre adolescente en el evento estudiado en esta investigación.

\section{CONCLUSIONES}

Con relación a la población con MME en Colombia en el tiempo estudiado, se pudo evidenciar que la población más afectada comprendía edades entre 15-29 y 30-44 años, dado que son mujeres en edad reproductiva y madres cabezas de familia, aspectos que pueden influir en el desarrollo de diferentes complicaciones; de igual forma, son rangos de edades muy amplios que aumentan en gran medida la problemática al no ser específicos, sino más bien generales, con lo cual se incrementa la probabilidad de que cualquier mujer pueda cursar por esta situación.

Así mismo, es de suma importancia resaltar que el mayor número de casos se presentó en el área urbana, razón por la que fue más fácil la recolección en las bases de datos de las entidades donde se atendieron a las pacientes. Esto, por otra parte, puede evidenciar un problema de sub-registro dado que las gestantes que presentan algún tipo de morbilidad deben acceder a los servicios de salud de alta complejidad en el área urbana, pues, en el área rural de Colombia, únicamente se cuenta con servicios de primer nivel, con los cuales no se puede realizar un tratamiento oportuno, eficaz e inmediato a estas poblaciones, razón que incrementa esta problemática.

Además de lo anterior, también se puede inferir que existen falencias en las estrategias para la 
prevención de estas enfermedades, pues son muchos los factores que pueden influir en la calidad de la educación que se les brinda a las gestantes; a lo dicho, se suma el hecho de que el seguimiento a estas pacientes no es eficaz $y$, de esta forma, se deja a la mujer en gestación y puerperio desprotegida.

Por otra parte, se observa un aumento significativo de la tasa de morbilidad; tal situación, puede estar relacionada con la falta de adherencia a las guías y programas de atención integral para esta población, lo que haría notable la necesidad de anticiparse al evento; así, se puede realizar para identificar las causas por medio de los datos estadísticos ya existentes. No se puede aceptar, por ningún motivo, la aparición de estos eventos en un país, pues este es un indicador que denota el interés por cumplir a cabalidad con los objetivos del milenio, que proponen cero muertes maternas y perinatales, pero esta problemática sigue causando muertes en los países.

En conclusión, la estimación de los indicadores de carga de la enfermedad, en especial los AVPP, evidencia necesidades poblacionales individualizadas que denotan la necesidad de que se ejecute una revisión exhaustiva de las políticas y programas para la prevención, manejo y seguimiento de los diferentes casos.

\section{AGRADECIMIENTOS}

Al Instituto Nacional de Salud, por su apoyo en la implementación y formación del SIVIGILA con el suministro de las bases de datos de Morbilidad Materna Extrema, y a la Universidad de Boyacá por sus contribuciones y ayudas metodológicas.

\section{CONFLICTOS DE INTERESES}

Las investigadoras del presente documento no refieren tener ningún tipo de conflicto de interés.

\section{REFERENCIAS}

1. Souza JP, Cecatti JG, Faundes A, Morais SS, Villar J, Carroli $G$ et al. Maternal near miss and maternal death in the World Health Organization's 2005 global survey on maternal and perinatal health. Bull World Heal Organ. 2010 [cited 2018 May 17];88:113-9. Available from: http://www.who.int/bulletin/volumes/88/2/08057828.pdf

2. Organización Mundial de la Salud. Evolución de la mortalidad materna: 1990-2015. Available from: http://apps.who.int/iris/bitstream/10665/204114/1/WHO_RHR_15.23_spa.pdf

3. Salazar Barrientos M. Factores asociados a la morbilidad materna extrema a nivel individual y entre las comunas y corregimientos 
de Medellín, 2013-2015: Repositorio Udea; 2017. Disponible en: http://tesis.udea.edu.co/ dspace/handle/10495/8800

4. Alkema L, Chou D, Hogan D, Zhang S, Moller $A B$, Gemmill A. Global, regional, and national levels and trends in maternal mortality between 1990 and 2015, with scenario-based projections to 2030: A systematic analysis by the Maternal Mortality Estimation Inter-Agency Group. Lancet. 2016;387:462-474. https:// doi.org/10.1016/S0140-6736(15)00838-7

5. Monroy AMM, Becerril GET, Vargas ÁG. Morbilidad materna extrema (near miss) y muertes maternas, Arch Inv Mat Inf. 2012;4(3):146-153

6. Tunçalp O, Hindin MJ, Souza JP, Chou D, Say L. The Prevalence of Maternal Near Miss. An Int J Obstet Gynaecol. 2012 Jun [cited 2018 Mar 10];119:653-61. https://doi.org/10.1111/ j.1471-0528.2012.03294.x

7. Cuesta MG. Morbilidad Materna Extrema (Near Miss) en el Hospital de la Mujer de Aguascalientes [Tesis Internet]. Universidad Autónoma de Aguascalientes 201. Disponible en: http://bdigital.dgse.uaa.mx:8080/xmlui/ handle/123456789/1434

8. Instituto Nacional de Salud. Protocolo de Vigilancia en Salud Pública. Morbilidad Materna
Extrema. Código 549; 2017. Disponible en: https://www.ins.gov.co/buscador-eventos/ ZIKA\%20Lineamientos/PRO\%20Morbilidad\%20materna\%20extrema_.pdf

9. Narváez Díaz NS. Informe del evento Morbilidad Materna Extrema, Colombia 2016. 2017.Disponible en: https://www.ins.gov.co/ buscador-eventos/Informesdeevento/Morbilidad\%20materna\%20extrema\%202016.pdf

10. Ministerio de Salud y Protección Social. Plan Decenal de Salud Pública 2012-2021 . 2012:452

11. Rolando E, Quintero N, Rodríguez J, García J, Beltrán A. Estimación de la carga de enfermedad para Colombia. Pontificia Universidad Javeriana; 2010:11-163. Disponible en: http://www. javeriana.edu.co/documents/12789/4434885/ Carga + de+Enfermedad+Colombia +2010 . pdf/e0dbfe7b-40a2-49cb-848e-bd67bf7bc62e

12. Murray CJ, Ezzati M, Flaxman $A D$, Lim $S$, Lozano R, Michaud C, Lopez AD. GBD 2010: Design, definitions, and metrics. The Lancet; 2012:380(9859), 2063-2066. https://doi. org/10.1016/S0140-6736(12)61689-4

13. Murray CJ, Lopez AD, World Health Organization, World Bank \& Harvard School of Public Health, editors. The Global Burden of Disease: a comprehensive assessment of mortality and disability from diseases, injuries, and 
risk factors in 1990 and projected to 2020: summary. Geneva: World Health Organization; 1996. Available from: http://www.who.int/iris/ handle/10665/41864

14. Bedregal P. Informe Final de Carga de Enfermedad y Carga Atribuible. Ministerio de Salud de Chile: 2008

15. Murray CJ, Ahmad O, Lopez AD, Salomon, JA. WHO System of Model Life Tables. GPE Discussion Paper Series. World Health Organization. 2003. Disponible en: https://www.who.int/ healthinfo/paper08.pdf

16. Peñaloza RE, Salamanca N, Rodríguez JM, Rodríguez J, Beltrán AR. Estimación de la carga de enfermedad para Colombia. Primera edición: Bogotá: Editorial Pontificia Universidad Javeriana; 2014. Disponible en: http://www.javeriana.edu.co/documents/12789/4434885/Car$\mathrm{ga}+$ de +Enfermedad + Colombia + 2010.pdf/ e0dbfe7b-40a2-49cb-848e-bd67bf7bc62e

17. Castañeda D, Martín G, Estévez A, Álvarez M, Salvador S. Caracterización de la morbilidad materna extremadamente grave en la provincia de Camagüey (2009). Rev Cubana Hig Epidemiol [Internet]. 2014;52(2):152-62. Disponible en: http://scielo.sld.cu/scielo.php?script=sci_arttext\&pid=S1561-30032013000100002
18. González J, Vázquez Y, Pupu J, Algas L, Cabrera JO. Morbilidad materna extrema en una unidad de cuidados intensivos Extreme maternal morbidity in an intensive care unit. MEDISAN [Internet]. 2015;19(12). Available from: http:// scielo.sld.cu/scielo.php?script =sci_arttext\&pi$d=$ S1029-30192015001200005\&lng =es\&nr$\mathrm{m}=$ iso\&tlng $=\mathrm{es}$

19. Bendezú G, Bendezú-Quispe G. Caracterización de la morbilidad materna extremadamente grave en un hospital del seguro social del Perú. Rev. peru. ginecol. obstet. [Internet]. 2014 Oct [citado 2018 Abr 13]; 60(4):291-298. Disponible en: http://www. scielo.org. pe/scielo.php? script $=$ sci_arttext\&pid=S2304-51322014000400004\&Ing=es.

20. Solórzano Chávez LM. Factores de riesgo asociados a la Morbilidad Materna Extrema en el Hospital Nacional Cayetano Heredia 2013 [internet]. Universidad Nacional Mayor de San Marcos; 2014. Disponible en: http://cybertesis.unmsm.edu.pe/bitstream/handle/cybertesis/4167/Solorzano_cl.pdf? sequence=1\&isAllowed $=\mathrm{y}$

21. Barbosa-Rengifo $M M$, Morales-Plaza CD, Amézquita-Abello MC, Martínez-Buitrago DM. Vigilancia de morbilidad materna extrema en una institución de referencia en Cali, Colombia 2013- 2014. Rev. Colombiana de Obstetricia y 
Ginecología [Internet] 2016;67(3):215-222 . https://doi.org/10.18597/rcog.769

22. Villalba-Toquica C, Martínez-Silva PA. Morbilidad materna extrema en la Clínica Universitaria Colombia, Bogotá (Colombia), 2012-2013. Revista Colombiana de Obstetricia y Ginecología [En línea], 65.4 (2014):290-296. https:// doi.org/10.18597/rcog.32

23. Carrillo Franco J, García Balaguera C. Comportamiento de la Morbilidad Materna Extrema en el Departamento del Meta, Colombia, 2014. Hacia promoc. salud. 2016; 21(1):15-25. http://dx.doi.org/10.17151/hpsal.2016.21.1.2

24. Donoso E, Carvajal JA, Vera C, Poblete JA. La edad de la mujer como factor de riesgo de mortalidad materna, fetal, neonatal e infantil. Rev. méd. Chile [Internet]. 2014 Feb [citado 2018 Nov 21]; 142(2):168-174. DOI: http://dx.doi. org/10.4067/S0034-98872014000200004.

25. Huang L, Sauve R, Birkett N, Fergusson D, van Walraven $C$. Maternal age and risk of stillbirth: a systematic review. CMAJ 2008; 178 (2): 165-72. https://doi.org/10.1503/cmaj.070150

26. Kenny LC, Lavender T, McNamee R, O'Neill SM, Mills T, Khashan AS. Advanced maternal age and adverse pregnancy outcome: evidence from a large contemporary cohort. PLoS One 2013;8(2):e56583. https://doi.org/10.1371/ journal.pone.0056583

27. Murray CJL, Lopez, AD. The utility of DALYs for public health policy and research: a reply; Bull World Health Organ. 1997;75(4):377-81. Available from: https://www.ncbi.nlm.nih.gov/ pubmed/9342897

28. Castañeda, C. N. Asociación de la carga de enfermedad por leucemia pediátrica aguda y las desigualdades en salud por régimen de afiliación y departamento de procedencia en Colombia [Tesis Maestría en Salud Pública]. Universidad Nacional de Colombia; 2016. 